\title{
One-Dimensional Analysis of Subthreshold Characteristics of SOI-MOSFET Considering Quantum Mechanical Effects
}

\author{
RIMON IKENO*, HIROSHI ITO and KUNIHIRO ASADA \\ Dept. of Electronic Eng., Fac. of Engineering, The University of Tokyo 7-3-1 Hongo, Bunkyo-ku, Tokyo 113, Japan
}

\begin{abstract}
We have been studying on subthreshold characteristics of SOI MOSFETs in terms of substrate bias dependence, using a 1-D Poisson equation on an SOI multi-layer structure for estimating structural parameters of real devices[1]. Here, we consider quantum mechanical effects in the electron inversion layer of thin SOI MOSFETs, implementing a self-consistent solver of Poisson and Schrödinger equations in a 1-D subthreshold simulator. From results of simulations, we have concluded that quantum mechanical effects need to be considered in analizing thin SOI devices.
\end{abstract}

Keywords: SOI MOSFET, device simulation, subthreshold characteristics, quantum mechanical effects, parameter fitting, substrate bias

\section{INTRODUCTION}

In SOI(Silicon-on-Insulator) devices, substrate bias has great influence on subthreshold characteristics of MOSFET's such as threshold voltage " $V_{t}$ " and subthreshold voltage swing " $\mathrm{S}$ ". Those characteristics are also affected by the structural device parameters of SOI multi-layers[2][3][4]. On the other hand, it is well-known that in thin electron inversion layer or thin silicon layer of SOI devices, 2-dimensional electron quantization effects should be taken into account [5].

In this study, we have implemented a self-consistent solver of Poisson and Schrödinger equations as a 1-D SOI MOSFET subthreshold device simulator and estimated device parameters by fitting simulated results with measured $V_{t h}-V_{B S}$ characteristics curves.

\section{ONE-DIMENSIONAL SIMULATION OF SOI MOSFET}

\section{Quantum Mechanical Modeling}

Figure 1 shows a schematic cross section of an SOI MOSFET. We solve following quantum mechanical models in the 1-D SOI multi-layer structure. Poisson equation (eq. 1) and Schrödinger equation (eq. 2) are solved self-consistently by the iterative method[6].

$$
\frac{d}{d x}\left\{\varepsilon \cdot \frac{d}{d x} \psi(x)\right\}=-q\left\{N_{A}-n(x)\right\}
$$

* Present address. Texas Instruments Tsukuba R\&D Center, 17 Miyukigaoka, Tsukuba, Ibaraki 305, Japan. Tel: +81-298-50-2668. Fax: +81-298-50-1729. E-mail: rimon@ti.com 


$$
\begin{gathered}
\left\{-\frac{\hbar^{2}}{2 m^{k}} \frac{d^{2}}{d x^{2}}+q \psi(x)\right\} \phi_{i}^{k}(x)=E_{i}^{k} \phi_{i}^{k}(x) \\
n(x)=\sum_{k=l, h} \sum_{i} N_{i}^{k} \cdot\left|\phi_{i}^{k}(x)\right|^{2} \\
N_{i}^{k}=n_{v}^{k} \cdot m_{d}^{k} \cdot k T \cdot \log \left[1+\exp \left\{\left(E_{F}-E_{i}^{k}\right) /(k T)\right\}\right] /\left(\pi \hbar^{2}\right)
\end{gathered}
$$

Here, $k$ denotes the lower or higher subband ladder; $l / h$, and $i$ denotes the subband index. $\phi_{i}{ }^{k}(x)$ is the electron wave function and $E_{i}{ }_{i}$ is the energy level for ith subband of ladder $k . n_{v}{ }^{k}$ is the valley degeneracies $\left(n_{v}{ }^{h}=2, \mathrm{n}_{v}{ }^{h}=4\right) . m^{l}\left(=0.98 m_{0} ; m_{0}\right.$ is the free electron mass) and $m^{h}\left(=0.19 m_{0}\right)$ are longitudinal and transverse mass of an electron. Then, $m_{d}{ }^{k}$ is formulated as follows: $m_{d}^{l}=m_{h}, m_{d}^{h}=\sqrt{ } m_{l} m_{h}$.

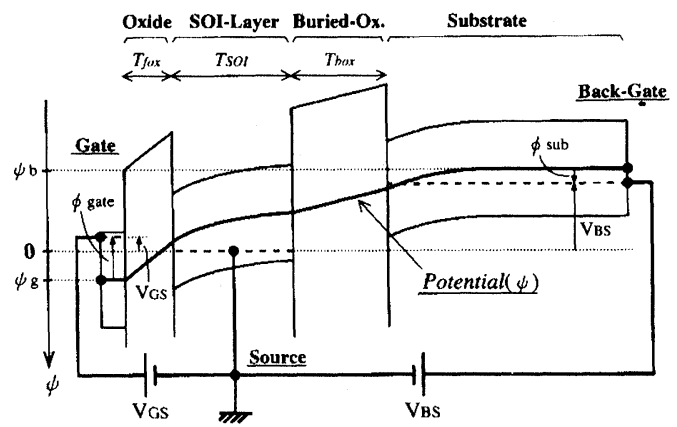

FIGURE 1 1-D device simulation of SOI MOSFET with front/back bias conditions

\section{Drain Current Modeling}

Drain current, $I_{d}$, is calculated as eq.5, which is composed of the diffusion current term. The drift current term is not taken into account because the major conduction mechanism is the carrier diffusion in subthreshold region.

$$
I_{d}=q W \frac{k T}{q} \mu \frac{n_{s}-n_{d}}{L}
$$

Here, $n_{d}$ and $n_{s}$ are channel carrier densities near source and drain, which are obtained by the 1-D simulation. We took into account thermal, ionized impurity and surface scattering effects for mobility, $\mu$.

\section{Comparison of Classical and Quantum-Mechanical Results}

Figure 2 shows distributions of electric potential and electron concentration in the SOI layer for both classical and quantum mechanical simulations. It is notable that the classical simulation gives the maximum of electron concentration at the front gate-oxide interface, while the quantum mechanical result shows zero at the interface. Figure 2 also shows a slight difference in the electric potential distributions. Drain currents are $1.95 \times 10^{-7}[A]$ for the classical simulation and $1.54 \times 10^{-7}[A]$ for the quantum mechanical simulation, for the device of $L=W=100 \mu \mathrm{m}$.

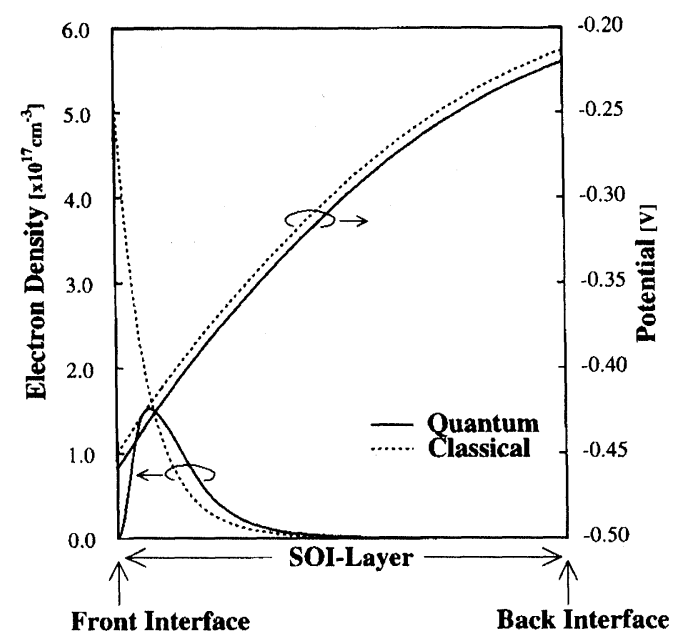

FIGURE 2 Simulated potential and electron concentration in SOI layer $\left(V_{B S}=0.0[\mathrm{~V}], V_{G S}=0.2[\mathrm{~V}]\right.$; solid-line: quantum mechanical, dotted-line: classical)

\section{DEVICE PARAMETER ESTIMATION}

We measured $V_{t h}-V_{B S}$ characteristics of SOI MOSFETs $\left(V_{t h} @ I_{d}=10 \mathrm{nA} / L=W=100 \mu \mathrm{m}\right)$. Then, we fitted the simulated curves with measured data, tuning device parameters such as channel dopant concentration, $N_{A}$, gate oxide thickness, $T_{f o x}$, SOI layer thickness, $T_{S O I}$, and buried oxide thickness, $T_{b o x}$.

Curve fitting procedure was carried out by the steepest descent method, evaluating a mean-square 
error of measured and simulated threshold voltages under various substrate bias conditions.

Figure 3 shows the best fitted curves along with measured data. The device parameters estimated from the curves are shown in Table I, with designed device parameters of real devices. The quantum mechanical model gives better results than the classical model in terms of extracted device parameters.

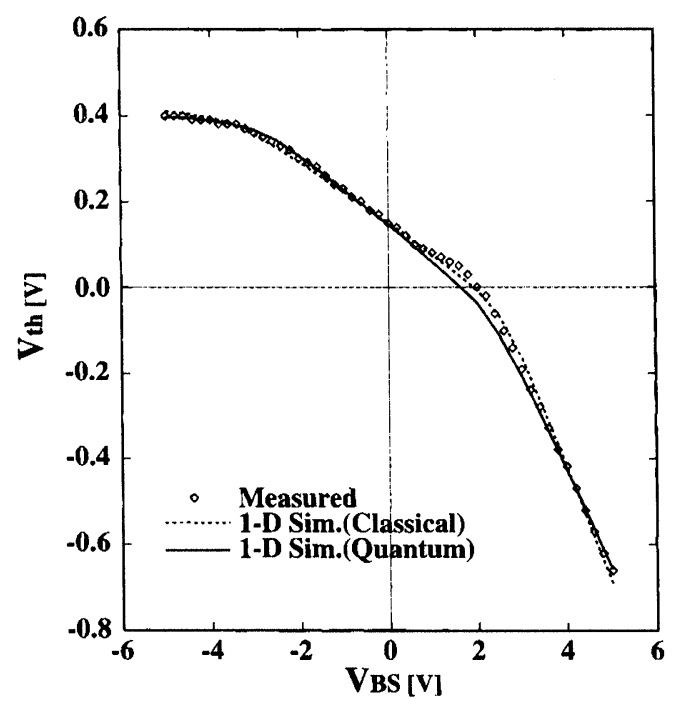

FIGURE 3 Vth-VBS characteristics of n-type SOI MOSFET, (by best-fitted parameters, $L=100 \mu \mathrm{m}, W=100 \mu \mathrm{m}$; solid-line:quantum mechanical sim., dotted-line: classical sim., points: measured)

TABLE I Designed and estimated device parameters

\begin{tabular}{llccc}
\hline & & Designed & Classical & Quantum \\
\hline$N_{A}$ & {$\left[10^{17} \mathrm{~cm}^{-3}\right]$} & - & 2.3 & 2.3 \\
$T_{f o x}$ & {$[\mathrm{~nm}]$} & 7 & 6.4 & 6.7 \\
$T_{S O I}$ & {$[\mathrm{~nm}]$} & 30 & 40 & 35 \\
$T_{b o x}$ & {$[\mathrm{~nm}]$} & 80 & 70 & 73 \\
\hline
\end{tabular}

\section{CONCLUSION}

A device simulator for 1-D SOI MOSFET subthreshold characteristics with a self-consistent Poisson and
Schrödinger equations solver was presented. Using the simulator, SOI device parameters were estimated by a curve fitting method for $V_{t h}-V_{B S}$ characteristics.

From the results, it was concluded that the quantum mechanical effects should be considered in thin SOI devices to obtain a better agreement with experimental data.

\section{References}

[1] R. Ikeno, H. Ito \& K. Asada, "Evaluation of SOI MOSFET Threshold Voltage using 1-D Device Simulation," Tech. Report of IEICE(in Japanese), SDM94-208, pp.25-30, Mar. 1995.

[2] D.J. Wouters, J.P. Colinge \& H.E. Maes, "Subthreshold Slope in Thin-Film SOI MOSFET's," IEEE Trans. Electron Devices, vol.17, no.9, pp.2022-2033, Nov. 1990.

[3] K. Tokunaga \& J.C. Sturm, "Substrate Bias Dependence of Subthreshold Slopes in Fully Depleted Siliconon-Insulator MOSFET's," IEEE Trans. Electron Devices, vol.38, no.8, pp.1803-1807, Aug. 1991.

[4] J.P. Colinge, "Subthreshold Slope of Thin-Film SOI MOSFET's," IEEE Electron Devices Letters, vol.7, no.4, pp.244, Apr. 1986.

[5] Y. Omura, S. Horiguchi \& K. Kishi, "Quantum-Mechanical Effects on the Threshold Voltage of Ultrathin- SOI nMOSFET'S," IEEE Electron Devices Letters, vol.14, no.12, pp.569-571, Dec. 1993.

[6] F. Stern, "Self-Consistent Results for n-Type Si Inversion Layers," Phys. Rev. B, vol.5, no.12, pp.4891-4899, June 1972.

\section{Biographies}

Rimon Ikeno is a doctor course student in electronic engineering, the University of Tokyo. His main works are modeling and simulation of small geometry semiconductor devices, and he is currently interested in SOI devices.

Hiroshi Ito is a doctor course student in electronic engineering, the University of Tokyo. He is interested in process integration and modeling of CMOS devices for low-power and high-speed application.

Kunihiro Asada is currently a Professor of Electronic Engineering Department in the School of Engineering of the University of Tokyo. His interest is in design and evaluation of integrated systems and their component devices. 

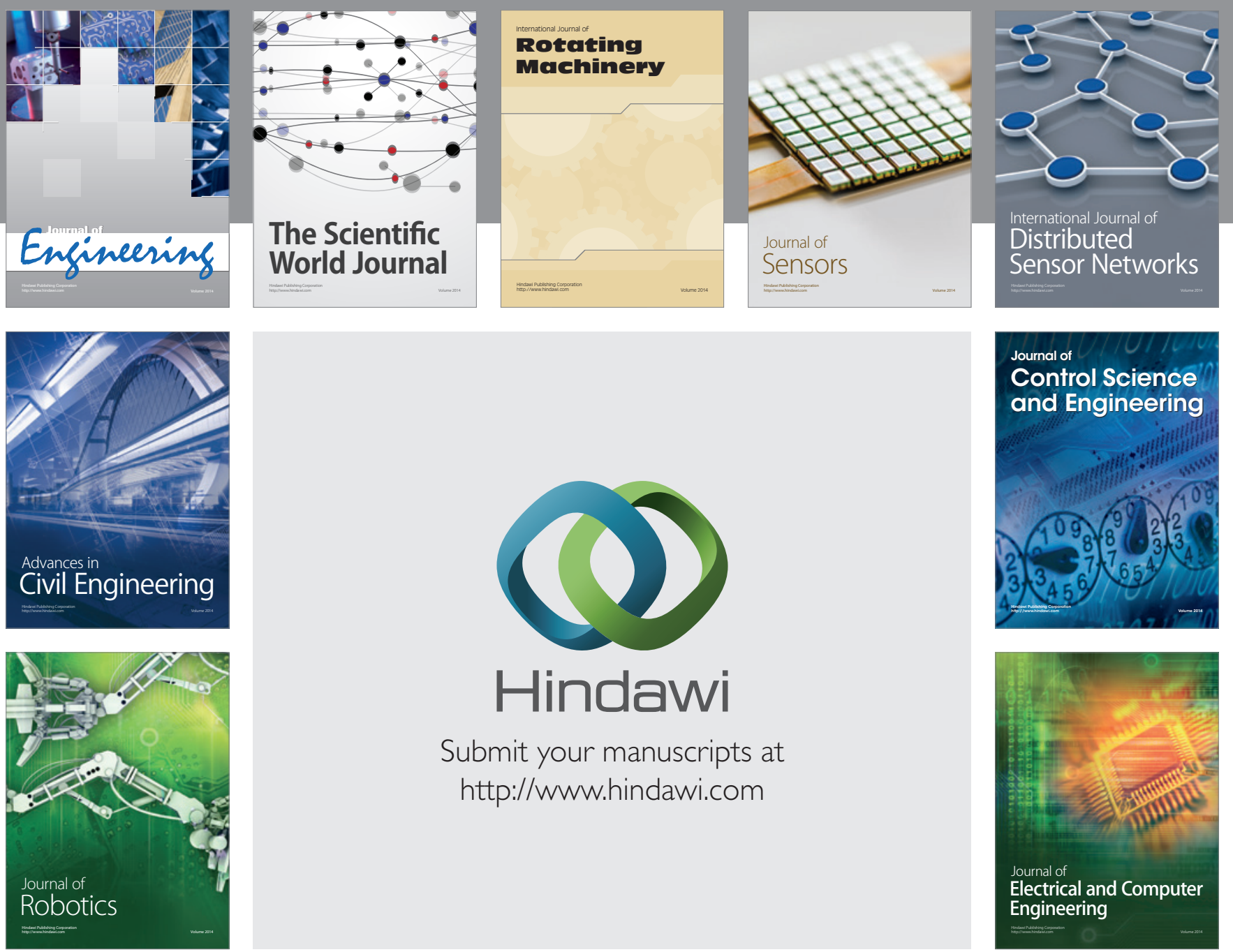

Submit your manuscripts at

http://www.hindawi.com
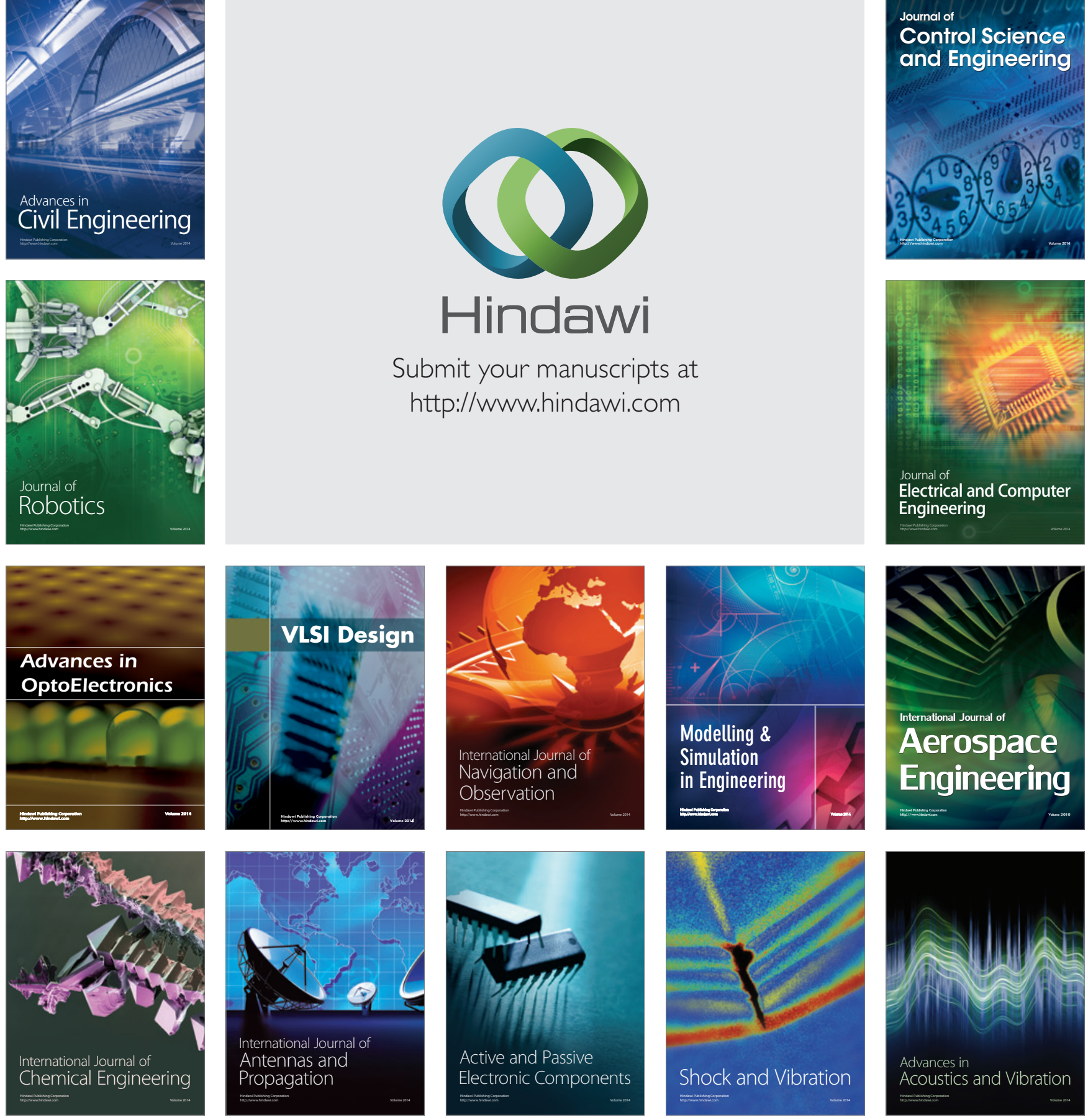